\title{
Imaging-Detected Incidental Thyroid Nodules that Undergo Surgery: A Single-Center Experience Over 1 Year
}

M. Bahl, J.A. Sosa, R.C. Nelson, and J.K. Hoang

\begin{abstract}
BACKGROUND AND PURPOSE: Incidental thyroid nodules are commonly seen on imaging, and their work-up can ultimately lead to surgery. We describe characteristics and pathology results of imaging-detected incidental thyroid nodules that underwent surgery.
\end{abstract}

MATERIALS AND METHODS: A retrospective review was performed of 303 patients who underwent thyroid surgery over a 1-year period to identify patients who presented with incidental thyroid nodules on imaging. Medical records were reviewed for the types of imaging studies that led to detection, nodule characteristics, and surgical pathology.

RESULTS: Of 303 patients, 208 patients (69\%) had surgery for thyroid nodules. Forty-seven of 208 patients (23\%) had incidental thyroid nodules detected on imaging. The most common technique leading to detection was CT (47\%). All patients underwent biopsy before surgery. The cytology results were nondiagnostic (6\%), benign (4\%), atypia of undetermined significance or follicular neoplasm of undetermined significance (23\%), follicular neoplasm or suspicious for follicular neoplasm (19\%), suspicious for malignancy (17\%), and diagnostic of malignancy (30\%). Surgical pathology was benign in 24 of $47(51 \%)$ cases of incidental thyroid nodules. In the 23 incidental cancers, the most common histologic type was papillary (87\%), the mean size was $1.4 \mathrm{~cm}$, and nodal metastases were present in 7 of 23 cases ( $30 \%)$. No incidental cancers on imaging had distant metastases.

CONCLUSIONS: Imaging-detected incidental thyroid nodules led to nearly one-fourth of surgeries for thyroid nodules, and almost half were initially detected on CT. Despite indeterminate or suspicious cytology results that lead to surgery, more than half were benign on final pathology. Guidelines for work-up of incidental thyroid nodules detected on CT could help reduce unnecessary investigations and surgery.

ABBREVIATIONS: FNAB $=$ fine needle aspiration biopsy; ITN $=$ incidental thyroid nodule

I ncidental thyroid nodules (ITNs) are commonly encountered on imaging studies, being seen in $50 \%$ of ultrasonographic studies and $16 \%-18 \%$ of CT and MR imaging studies that include the thyroid gland. ${ }^{1-3}$ While ITNs are associated with a low rate of malignancy, and subclinical thyroid cancers have an excellent prognosis, ${ }^{2-6}$ ITNs pose a management dilemma for radiologists and other clinicians whose concern for missing malignancies may lead to further evaluation for small nonspecific thyroid nodules.

Received March 5, 2014; accepted after revision April 16

From the Departments of Radiology (M.B., R.C.N., J.K.H.), Radiation Oncology (J.K.H.), and Surgery, Duke Cancer Institute and Duke Clinical Research Institute (J.A.S.), Duke University Medical Center, Durham, North Carolina.

Paper previously presented at: Annual Meeting of the American Society of Head and Neck Radiology, September 25-39, 2013; Milwaukee, Wisconsin.

M. Bahl was the recipient of the ASHNR Radiologist-in-Training Award for 2013.

Please address correspondence to Jenny K. Hoang, MBBS, Duke University Medical Center, Department of Radiology, Box 3808, Erwin Rd, Durham, NC 27710; e-mail: jennykh@gmail.com

http://dx.doi.org/10.3174/ajnr.A4004
The reporting practice of ITNs seen on imaging is highly variable among radiologists. ${ }^{7}$ Radiologists must exercise their judgment when reporting and issuing recommendations regarding incidental thyroid nodules. The reporting of ITN on imaging can lead to further investigation, such as follow-up sonography examinations, fine needle aspiration biopsy (FNAB), or, in some cases, diagnostic thyroid lobectomy or thyroidectomy. ${ }^{8,9}$ Of patients who undergo FNAB, 22\%-51\% proceed to surgery. ${ }^{10-12}$ These surgical patients represent an important group to study because they have higher costs and morbidity associated with the work-up of their ITNs. Thus, it is important to consider the costs and benefits of work-up in patients with ITN who fall into this surgical group.

A substantial number of patients who undergo surgery do not have cancer. ${ }^{10}$ When the preoperative cytology result from FNAB is malignant, the sensitivity of cytology is high (99\%). ${ }^{13}$ However, it is recommended that patients with cytology of "follicular neoplasm," "suspicion for follicular neoplasm," and "suspicion for malignancy" also proceed to surgery. With these other categories, the false-positive rate of cytology can be as high as $44 \% .{ }^{13}$ In 
addition, thyroid surgery can result in such complications as recurrent laryngeal nerve injury, hypoparathyroidism, and bleeding. Therefore, radiologists should understand the downstream sequelae of a clinical pathway that begins with an imaging-detected ITN and ends with surgery.

The purpose of this study was to describe characteristics and pathology results of ITNs that undergo surgery. We hypothesize that imaging-detected ITN comprise a substantial proportion of surgeries for thyroid nodules, and that many thyroid nodules are in the end benign on final surgical pathology.

\section{MATERIALS AND METHODS Study Subjects}

We performed a retrospective study of 303 consecutive patients who underwent thyroidectomy or lobectomy between January 1 , 2012, and December 31, 2012, at a single large academic institution to identify patients who presented with an ITN on imaging. Patients were identified by Current Procedural Terminology codes for thyroid lobectomy and thyroidectomy (60210, 60212, 60220, 60240, 60252, 60254, 60255, 60260, 60270, and 60271). Medical records were reviewed for the presentation of the thyroid nodule and identification of thyroid cancer. This study was approved by the Institutional Review Board and was compliant with the Health Insurance Portability and Accountability Act.

An ITN on imaging was defined as a nodule originally detected on an imaging study performed for other reasons in a patient with no clinical symptoms, examination findings, or suspicion for thyroid cancer. Patients were excluded if they had a palpable nodule or mass (representing primary or nodal disease) or had thyroid nodules detected on sonography performed for abnormal thyroid function test results without a palpable nodule or mass. The decision to biopsy an ITN is made by the referring clinician. Many clinicians follow the American Thyroid Association guidelines for sonography criteria, but this may not be uniform practice. ${ }^{14}$

Patients with ITN generally proceed to surgery if the cytologic results obtained by FNAB demonstrate follicular neoplasm or suspicion for follicular neoplasm, suspicion for malignancy, or malignancy (Bethesda classes IV, V, and VI). ${ }^{15}$ Patients could also proceed to surgery if cytology demonstrates atypia of undetermined significance or follicular lesion of undetermined significance (Bethesda class III) if they do not want to undergo surveillance or rebiopsy or have worrisome characteristics on sonography. Medical records were also reviewed for demographic information, including patient age and sex, clinical features, including the risk factors for thyroid cancer such as a family history of glandular abnormalities or exposure to external beam radiation, and nodule characteristics on imaging.

\section{Outcome Measures and Statistical Analysis}

The primary outcomes of interest were the cytology and final surgical pathology results. These results were interpreted by board-certified pathologists. We also described the pattern of work-up, including imaging studies leading to detection, subsequent steps in work-up, and timing of surgery after detection of the nodule. The imaging-detected incidental cancers were reviewed for cancer characteristics, histology, and tumor stage according to the American Joint Committee on Cancer. ${ }^{16}$ Patient

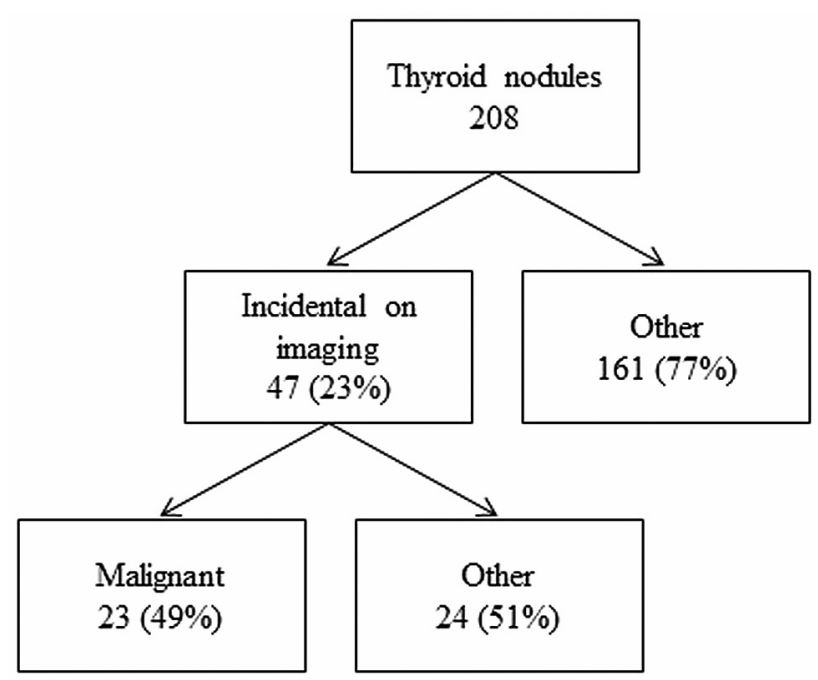

FIG 1. Flowchart of study subjects.

demographic data, nodule size, imaging modalities, and FNAB cytology were compared for the benign and malignant imaging-detected ITNs with the unpaired $t$-test for continuous data and the $\chi^{2}$ test or Fisher exact test for categoric data. The Fisher exact test was used when expected cell frequencies were less than 5 . Specifically, for categoric variables with independent groups, $P$ values were calculated for CT versus other imaging modalities and for cytology diagnostic categories I, II, and III versus IV, V, and VI. $P<.05$ was considered to indicate a statistically significant difference. Data were entered into a spreadsheet for analysis and generation of the descriptive statistics (Excel; Microsoft, Redmond, Washington). Statistical analyses were performed using SAS Enterprise software (version 4.2; SAS Institute, Cary, North Carolina).

\section{RESULTS}

Over a 1-year period, 303 patients (236 females and 67 males, mean age 51 years, age range of $8-87$, and with 5 patients younger than 16 years of age) underwent thyroid surgery for symptomatic and asymptomatic thyroid disease. Of the 303 patients who underwent surgery, 208 (69\%) patients underwent surgery for thyroid nodules and 95 (31\%) were for symptomatic multinodular goiter or hyperthyroidism refractory to medical treatment (Fig 1).

Of the 208 patients who presented with thyroid nodules, 47 $(23 \%)$ had ITNs detected on imaging (Table 1). The most common imaging studies leading to detection were CT (22 of 47, $47 \%$ ), followed by PET/CT ( 10 of 47, 21\%), sonography ( 9 of 47 , $19 \%$ ), MR imaging ( 4 of $47,9 \%$ ), chest radiograph ( 1 of $47,2 \%$ ), and echocardiogram ( 1 of 47, 2\%). Of note, 6 of the sonography studies were performed for evaluation of the carotid arteries, and 3 were performed for purposes of parathyroid adenoma localization in patients with primary hyperparathyroidism. All 47 patients with imaging-detected ITN underwent FNAB within a median of 34 days (interquartile range 18-82 days). In 4 (9\%) patients, the decision to biopsy was based on the initial imaging, and full diagnostic sonography was not performed.

The FNAB cytology results for the 47 patients with imagingdetected ITN were nondiagnostic $(n=3,6 \%)$, benign $(n=2$, $4 \%$ ), atypia of undetermined significance or follicular neoplasm of undetermined significance ( $n=11,23 \%)$, follicular neoplasm 
Table 1: Characteristics of benign and malignant ITNs that undergo surgery

\begin{tabular}{|c|c|c|c|c|}
\hline & $\begin{array}{l}\text { Benign } \\
\text { ITN }\end{array}$ & $\begin{array}{l}\text { Malignant } \\
\text { ITN }\end{array}$ & $\begin{array}{l}\text { All } \\
\text { ITN }\end{array}$ & $\begin{array}{c}P \\
\text { Value }\end{array}$ \\
\hline $\mathrm{Age}^{\mathrm{a}}$ & $58 \pm 14$ & $54 \pm 13$ & $56 \pm 14$ & .3 \\
\hline \multicolumn{5}{|l|}{ Sex } \\
\hline Female & $16(67)$ & $19(83)$ & $35(74)$ & $.3^{\mathrm{b}}$ \\
\hline Male & $8(33)$ & $4(17)$ & $12(26)$ & \\
\hline \multicolumn{5}{|l|}{ Nodule size (in mm) } \\
\hline Imaging $^{\mathrm{a}}$ & $22 \pm 13$ & $17 \pm 7$ & $20 \pm 11$ & .2 \\
\hline Pathology & NA & $14 \pm 8$ & NA & \\
\hline Imaging modality on which ITN was detected & & & & $.2^{\mathrm{b}, \mathrm{c}}$ \\
\hline CT & $9(38)$ & $13(57)$ & $22(47)$ & \\
\hline $\mathrm{PET} / \mathrm{CT}$ & $4(17)$ & $6(26)$ & $10(21)$ & \\
\hline Ultrasound & $7(29)$ & $2(9)$ & 9 (19) & \\
\hline MRI & $3(13)$ & $1(4)$ & $4(9)$ & \\
\hline Chest radiograph & $1(4)$ & 0 & $1(2)$ & \\
\hline Echocardiogram & 0 & $1(4)$ & $1(2)$ & \\
\hline Cytology & & & & $<.001^{\mathrm{b}, \mathrm{d}}$ \\
\hline I-Nondiagnostic or unsatisfactory & $2(8)$ & $1(4)$ & $3(6)$ & \\
\hline II—Benign & $2(8)$ & 0 & $2(4)$ & \\
\hline $\begin{array}{l}\text { III-Atypia of undetermined significance/ } \\
\text { follicular lesion of undetermined significance }\end{array}$ & $10(42)$ & $1(4)$ & $11(23)$ & \\
\hline $\begin{array}{l}\text { IV_Follicular neoplasm/suspicious for } \\
\text { follicular neoplasm }\end{array}$ & $8(33)$ & $1(4)$ & $9(19)$ & \\
\hline V—Suspicious for malignancy & $2(8)$ & $6(26)$ & $8(17)$ & \\
\hline VI_Malignant & 0 & $14(61)$ & $14(30)$ & \\
\hline Total & 24 & 23 & 47 & \\
\hline
\end{tabular}

Note:- Unless otherwise specified, data are numbers of patients, with percentages in parentheses. Percentages may not add up to $100 \%$ due to rounding.

a Data reported as mean \pm standard deviation.

${ }^{\mathrm{b}}$ Calculated with Fisher exact test.

${ }^{c}$ For $\mathrm{CT}$ versus other imaging modalities.

${ }^{\mathrm{d}}$ For diagnostic categories I, II, and II versus IV, V, and VI.

or suspicious for follicular neoplasm $(n=9,19 \%)$, suspicious for malignancy $(n=8,17 \%)$, and diagnostic of malignancy $(n=14$, $30 \%)$. Note that the 2 patients with benign cytologies underwent thyroid lobectomy at the same time as parathyroidectomy for primary hyperparathyroidism.

The median time between the FNAB and thyroid surgery was 46 days (interquartile range 28-77 days). Despite the cytology results, the final surgical pathology was benign in 24 of 47 (51\%). In the 23 incidental cancers, the histologic types were 20 papillary (87\%), 2 follicular (9\%), and 1 medullary (4\%) thyroid cancer. The mean and median size of imaging-detected tumors was 1.4 $\mathrm{cm}(\mathrm{SD} 0.8 \mathrm{~cm}$ ) and $1.2 \mathrm{~cm}$ (interquartile range $1.0-1.6 \mathrm{~cm}$ ), respectively. Nodal metastases were present in 7 of 23 (30\%), 4 of which were present in the central neck compartment (N1a) and 3 of which were present in the lateral neck compartment (N1b). No incidental cancers on imaging had distant (extra-cervical) metastases.

\section{DISCUSSION}

Outcomes of imaging-detected ITN have been reported, but this study is the first to focus on ITN in patients who underwent surgery. This group represents only a fraction of all ITNs encountered in a radiology practice, but it is a group that is more likely to harbor malignancy and to have higher costs associated with workup. We found that imaging-detected ITNs comprise nearly onefourth of surgeries for thyroid nodules. Despite cytology results that lead to surgery, more than half of ITNs are ultimately benign on final surgical pathology.
The malignancy rate in ITNs undergoing surgery varies widely in the existing literature. Kroeker et al ${ }^{11}$ studied $133 \mathrm{im}$ aging-detected ITNs referred to a single surgeon and found that $41 \%$ were managed surgically, of which $86 \%$ were malignant. Hobbs et al ${ }^{10}$ also evaluated 114 imaging-detected ITNs in patients who underwent FNAB under sonography guidance. They found that $25 \%$ of patients proceeded to surgery after FNAB, and only $24 \%$ of these patients were diagnosed with thyroid cancer. The marked difference in malignancy rate is likely related to differences in the study cohorts. Patients referred to a single surgeon in Kroeker et al's ${ }^{11}$ study were more likely to have malignancy because they were referred for concerning features that warrant surgery. Hobbs et al's ${ }^{10}$ study cohort was more likely to have benign pathology results because it represents indeterminate ITNs that a radiologist was asked to biopsy under sonography, and many of these nodules were small. Our study had a malignancy rate of $49 \%$, which lies between these 2 studies. All 3 studies highlight that FNAB is a minimally invasive and critical diagnostic tool, but it is important to select nodules carefully for biopsy. After FNAB, a significant portion of patients with imagingdetected ITN proceed to surgery because of suspicious or indeterminate cytology results and many may be for benign disease. New strategies, such as sonography elastography and molecular testing, may reduce unnecessary surgery in those patients with inconclusive FNAB. ${ }^{17}$ Another strategy may be to apply stricter guidelines to reduce FNAB or to observe thyroid nodules with serial ultrasounds rather than performing FNAB. ${ }^{14,18}$

Another reason for studying a group of surgical ITNs is to understand the contribution of imaging-detected ITN to a surgical cohort. In our study, $23 \%$ of thyroid nodules that proceeded to surgery were imaging-detected ITNs. The rate of ITN is similar to the study by Hobbs et al ${ }^{10}$ whose cohort were patients undergoing sonography-guided FNAB; they found that 29\% (114 of 390) of these patients presented with imaging-detected ITN. Other studies that focus on thyroid cancers report that 15\%-39\% are incidental cancers detected on imaging. ${ }^{19,20}$ It is possible that many of the incidental cases in our study would not have been diagnosed in the patient's lifetime if not for imaging. If we consider the scenario in which they were not diagnosed, the impact would be 23 undiagnosed cancers and 24 patients who would not have undergone work-up and surgery for benign disease in 1 year at our institution. While missing 23 cancers in 1 year may alarm some radiologists, it is important to recognize that most of the cancers were localized small papillary thyroid cancers, which have excellent prognosis with treatment. Although it is controversial, many believe the prognosis is excellent even without treatment, especially for older patients ( $\geq 60$ years). ${ }^{21,22}$ The disadvantage of 
Table 2: The Duke 3-tiered system for CT, MRI, or PET-detected thyroid nodules

\begin{tabular}{|c|c|c|}
\hline Category & Criteria for Categories & Recommendations \\
\hline $\begin{array}{l}\text { Risk category 1: Highly suspicious } \\
\text { for malignancy }\end{array}$ & $\begin{array}{l}\text { PET avid thyroid nodule } \\
\text { Suspicious lymphadenopathy }{ }^{\text {a }} \\
\text { Extrathyroid spread with or without signs of } \\
\quad \text { vocal cord palsy on side of nodule } \\
\text { Lung metastases }\end{array}$ & $\begin{array}{l}\text { Strongly consider work-up with ultrasound } \\
\text { for any size nodule }\end{array}$ \\
\hline $\begin{array}{l}\text { Risk category 2: Indeterminate with } \\
\text { risk factor of young age }\end{array}$ & Age $<35$ years & $\begin{array}{l}\text { Consider work-up with ultrasound if } \geq 1 \mathrm{~cm} \text { in adults } \\
\text { Consider work-up with ultrasound for any size } \\
\text { in pediatric patients }\end{array}$ \\
\hline $\begin{array}{l}\text { Risk category 3: Indeterminate } \\
\text { without risk factors }\end{array}$ & Age $\geq 35$ years & Consider work-up with ultrasound if $\geq 1.5 \mathrm{~cm}$ \\
\hline
\end{tabular}

Note:-Intended for management of incidental thyroid nodules in low-risk patients.

${ }^{\text {a }}$ Suspicious lymph nodes are defined as nodes $>10 \mathrm{~mm}$ in short axis (with the exception of jugulodigastric lymph nodes, which were permitted to be up to $15 \mathrm{~mm}$ in short axis), or nodes that contained either calcifications, cystic components, or irregular margins.

work-up is that these 47 patients represent just a small fraction of the patients who underwent work-up for ITN with sonography and FNAB. The health care costs attributed to work-up of thyroid nodules is significant because 1 in 2 Americans have thyroid nodules greater than $5 \mathrm{~mm} .^{6}$

An unexpected finding in this study was that CT accounted for almost half of ITNs that went to surgery. Sonography has higher resolution than CT and has been commonly proposed as the primary technique responsible for the over-diagnosis of thyroid cancer. ${ }^{23}$ Our study draws attention to CT as a large source of ITNs. This finding may also be explained, in part, by the fact that CT utilization has increased rapidly in the United States, at a rate greater than sonography, and utilization of CT scanning exceeds sonography. ${ }^{24,25}$ Hoang et $\mathrm{al}^{24}$ reported a strong linear relationship between new cases of subcentimeter thyroid cancer and the number of CT scans per year, suggesting that the increase in CT scans may account for increased detection of incidental thyroid cancers. The fact that nearly half of ITNs that proceed to surgery are detected on CT underscores the need for guidelines to reduce the potentially unnecessary work-up of CT-detected ITN. The Duke three-tiered system (Table 2), for example, addresses ITN on CT, MR imaging, and PET/CT and has been shown to reduce the number of benign biopsies by more than one-third without missing clinically significant malignancies. ${ }^{3,10,26}$

There were several limitations to this study. First, this study was retrospective in nature and was performed at a single academic radiology practice, which may differ from other institutions with regard to the reporting and work-up of incidental thyroid nodules, the diagnostic yield of FNAB, and the accuracy of cytopathology. Second, the criteria we used to define incidental cancers on imaging may underestimate the incidental cancer rate compared with criteria used in other studies. If we had included nonpalpable cancers in patients with abnormal thyroid function test results, the prevalence of incidental thyroid cancers would be higher. We chose to exclude these groups to estimate the impact of work-up of nodules that radiologists encounter when imaging is performed for reasons truly unrelated to the thyroid gland.

\section{CONCLUSIONS}

Imaging-detected ITNs comprise nearly one-fourth of surgeries for thyroid nodules at our institution, and CT scans are the source of almost half of the ITNs identified in this series. Despite indeterminate or suspicious cytology that leads to surgery, more than half are benign on final pathology. Most incidental thyroid can- cers on imaging are small papillary cancers without nodal and distant metastases. Future guidelines that address reporting and work-up of ITNs seen on CT imaging may better tailor work-up and reduce unnecessary surgery.

Disclosures: Julie Sosa-UNRELATED: participated in one-time advisory committee meeting sponsored by Bayer; received one-time honorarium from Exelixis for a speech about surgery. Rendon Nelson-UNRELATED: Consultancy: GE Healthcare; Expert Testimony: settled case Woessner v. Toledo Hospital; Grants/Grants Pending: Bracco Diagnostics for research support*; Payment for Lectures Including Service on Speakers Bureaus: Applied Radiology; Patents Issued: GE Healthcare, Comments: using the scout radiograph on CT to determine the contrast media dose (no payment or money for this); Royalties: Lippincott Williams and Wilkins. *Money paid to the institution.

\section{REFERENCES}

1. Acar T, Ozbek SS, Acar S. Incidentally discovered thyroid nodules: frequency in an adult population during Doppler ultrasonographic evaluation of cervical vessels. Endocrine 2014;45:73-78

2. Yousem DM, Huang T, Loevner LA, et al. Clinical and economic impact of incidental thyroid lesions found with CT and MR. AJNR Am J Neuroradiol 1997;18:1423-28

3. Nguyen XV, Choudhury KR, Eastwood JD, et al. Incidental thyroid nodules on CT: evaluation of 2 risk-categorization methods for work-up of nodules. AJNR Am J Neuroradiol 2013;34:1812-17

4. Yoon DY, Chang SK, Choi CS, et al. The prevalence and significance of incidental thyroid nodules identified on computed tomography. J Comput Assist Tomogr 2008;32:810-15

5. National Cancer Institute Surveillance. Epidemiology, and End Results, Data 1983 to 2009. http://seer.cancer.gov/. Accessed December 15,2012

6. Smith-Bindman R, Lebda P, Feldstein VA, et al. Risk of thyroid cancer based on thyroid ultrasound imaging characteristics: results of a population-based study. JAMA Intern Med 2013;173:1788-96

7. Hoang JK, Riofrio A, Bashir MR, et al. High variability in radiologists' reporting practices for incidental thyroid nodules detected on CT and MRI. AJNR Am J Neuroradiol 2014;35:1190-94

8. Centers for Medicare and Medicaid Services. Physician Fee Schedule Search. http://www.cms.gov/apps/physician-fee-schedule/. Accessed December 4, 2012

9. Bahl M, Sosa JA, Nelson RC, et al. Thyroid cancers incidentally detected at imaging in a 10 -year period: how many cancers would be missed with use of the recommendations from the Society of Radiologists in Ultrasound? Radiology 2014;271:888-94

10. Hobbs HA, Bahl M, Nelson RC, et al. JOURNAL CLUB: Incidental thyroid nodules detected at imaging: can diagnostic workup be reduced by use of the Society of Radiologists in Ultrasound recommendations and the three-tiered system? AJR Am J Roentgenol 2014;202:18-24

11. Kroeker TR, le Nobel G, Merdad M, et al. Outcomes of incidentally 
discovered thyroid nodules referred to a high-volume head and neck surgeon. Head Neck 2014;36:126-29

12. Shrestha M, Crothers BA, Burch HB. The impact of thyroid nodule size on the risk of malignancy and accuracy of fine-needle aspiration: a 10-year study from a single institution. Thyroid 2012;22:1251-56

13. Bongiovanni M, Spitale A, Faquin WC, et al. The Bethesda system for reporting thyroid cytopathology: a meta-analysis. Acta Cytol 2012;56:333-39

14. American Thyroid Association (ATA) Guidelines Taskforce on Thyroid Nodules and Differentiated Thyroid Cancer, Cooper DS, Doherty GM, et al. Revised American Thyroid Association management guidelines for patients with thyroid nodules and differentiated thyroid cancer. Thyroid 2009;19:1167-214

15. Cibas ES, Ali SZ. NCI Thyroid FNA. State of the Science Conference. The Bethesda system for reporting thyroid cytopathology. Am J Clin Pathol 2009;132:658-65

16. National Cancer Institute. Stage Information for Thyroid Cancer. http:// www.cancer.gov/cancertopics/pdq/treatment/thyroid/HealthProfessional/ page3. Accessed December 6, 2012

17. Tonacchera M, Pinchera A, Vitti P. Assessment of nodular goitre. Best Pract Res Clin Endocrinol Metab 2010;24:51-61

18. Frates MC, Benson CB, Charboneau JW, et al. Management of thy- roid nodules detected at US: Society of Radiologists in Ultrasound consensus conference statement. Radiology 2005;237:794-800

19. Bahl M, Sosa JA, Nelson RC, et al. Trends in incidentally identified thyroid cancers over a decade: a retrospective analysis of 2,090 surgical patients. World J Surg 2014;38:1312-17

20. Malone MK, Zagzag J, Ogilvie JB, et al. Thyroid cancers detected by imaging are not necessarily small or early stage. Thyroid 2014; 24:314-18

21. Ito $\mathrm{Y}$, Miyauchi $\mathrm{A}$, Inoue $\mathrm{H}$, et al. An observational trial for papillary thyroid microcarcinoma in Japanese patients. World J Surg 2010;34:28-35

22. Ito $\mathrm{Y}$, Miyauchi A, Kihara M, et al. Patient age is significantly related to the progression of papillary microcarcinoma of the thyroid under observation. Thyroid 2014;24:27-34

23. Davies L, Welch HG. Increasing incidence of thyroid cancer in the United States, 1973-2002. JAMA 2006;295:2164-67

24. Hoang JK, Choudhury KR, Eastwood JD, et al. An exponential growth in incidence of thyroid cancer: trends and impact of CT imaging. AJNR Am J Neuroradiol 2014;35:778-83

25. Davies L, Welch HG. Current thyroid cancer trends in the United States. JAMA Otolaryngol Head Neck Surg 2014;140:317-22

26. Hoang JK, Raduazo P, Yousem DM, et al. What to do with incidental thyroid nodules on imaging? An approach for the radiologist. Semin Ultrasound CT MR 2012;33:150-57 